\title{
Acknowledgement to Reviewers of Vaccines in 2016
}

\author{
Vaccines Editorial Office, \\ Published: 10 January 2017 \\ MDPI AG, St. Alban-Anlage 66, 4052 Basel, Switzerland; vaccines@mdpi.com
}

The editors of Vaccines would like to express their sincere gratitude to the following reviewers for assessing manuscripts in 2016.

We greatly appreciate the contribution of expert reviewers, which is crucial to the journal's editorial process. We aim to recognize reviewer contributions through several mechanisms, of which the annual publication of reviewer names is one. Reviewers receive a voucher entitling them to a discount on their next MDPI publication and can download a certificate of recognition directly from our submission system. Additionally, reviewers can sign up to the service Publons (https://publons.com) to receive recognition. Of course, in these initiatives we are careful not to compromise reviewer confidentiality. Many reviewers see their work as a voluntary and often unseen part of their role as researchers. We are grateful to the time reviewers donate to our journals and the contribution they make.

If you are interested in becoming a reviewer for Vaccines, see the link at the bottom of the webpage http://www.mdpi.com/reviewers.

The following reviewed for Vaccines in 2016:

Austin, Brian
Ayalew, Sahlu
Bachmann, Martin
Banoub, Joseph H.
Barnier-Quer, Christophe
Baud, David
Berkhout, Ben
Binley, James
Bogers, Willy M.J.M.
Boscardin, Silvia
Bradford, Leslie S.
Bultema, Jarred J.
Chatel, Jean-Marc
Chen, Shih-Chu
Chlichlia, Katerina
Christodoulides, Myron
Church, Sarah
Clementi, Emilio
Cortez, Maria Angelica
Cuenda, Ana
Currie, Margaret
Daniels-Wells, Tracy R.
De Pascale, Donatella
DeLong, Robert K.
Demchenko, Alexei
Eko, Francis

El-Matbouli, Mansour
Epstein, Alan
Ferrone, Soldano
Fondi, Marco
Fontaine, Magali J.
Frelin, Lars
Fröhlich, Eleonore
Fujimori, Ko
Geary, Sean
Getts, Daniel R.
Goedegebuure, Peter
Goepfert, Paul
Grivennikov, Sergei I.
Gruber, Christian
Hall, Pamela
Halle, Stephan
Haller, Otto
Henao-Tamayo, Marcela I.
Heng, Tracy
HogenEsch, Harm
Holst, Johan
Huang, Xuefei
Hudalla, Gregory A.
Iwamori, Masao
Jangra, Rohit
Jones, Harlan P.

\author{
Kamachi, Kazunari \\ Koumoto, Kazuya \\ Krauss, Isaac J. \\ Kumar, Gokhlesh \\ LaJoie, A. Scott \\ Lathia, Justin \\ Liu, Zhixia \\ Lomonossoff, George P. \\ Mahony, Timothy John \\ Maita, Masashi \\ Majlessi, Laleh \\ Mathew, R.O. \\ Matysiak, Jan \\ McArdle, Stephanie \\ McClean, Siobhan \\ Mehrotra, Shikhar \\ Melillo, Rosa Marina \\ Moreland, Nikki \\ Morrison, Trudy G. \\ Moutsopoulos, Haralampos M. \\ Müller-Nordhorn, Jacqueline \\ Mullins, David W. \\ Murthy, Ashlesh K. \\ Nativi, Cristina \\ Nayak, Jennifer L. \\ Nebreda, Angel R.
}


Nguyen, Andrew V.

Nijnik, Anastasia

Nishimura, Shin-ichiro

Orzechowski, Arkadiusz

Paes, Wayne

Pancholi, Vijay

Peeples, Mark

Piedra, Pedro A.

Puentes, Fabiola

Rolfe, Barbara E.

Rutkowski, Melanie

Saelens, Xavier

Santosham, Mathuram

Sastry, Jagan

Sauerwein, Robert W.

Savage, Paul B.

Schicho, Rudolf

Schiller, John

Schmitt, Egdar

Schroit, Alan
Scott, Nick

Shrikant, Protul

Skwarczynski, Mariusz

Smerdou, Cristian

Snapp, Erik Lee

Sommerer, Claudia

Sounni, Nor Eddine

Striegler, Susanne

Suzuki, Yasuhiro

Tan, Tina Q.

Tartour, Eric

Tavernier, Jan

Tenbusch, Matthias

Theisen, Michael

Thornburg, Natalie

Trilling, Mirko,

Ueno, Hideki

Ulbert, Sebastian

Umansky, Viktor

Valvano, Miguel A.
Van De Ven, Rieneke

Van Griensven, Leo J.L.D

Veazey, Ronald

Verbrugge, Inge

Villalba, Martin

Wang, Jun

Wang, Ying

Wesolowski, Robert

Whiteside, Theresa

$\mathrm{Wu}$, Xueling

Wunderlich, Thomas

Xin, Hong

$\mathrm{Xu}$, Zhiping

Youn, Je-In

$\mathrm{Yu}$, Chengzhong

Zhang, Peipei

Zhang, Qibo

Zhu, Yuwen

Zimmer, Gert

(C) 2017 by the authors; licensee MDPI, Basel, Switzerland. This article is an open access article distributed under the terms and conditions of the Creative Commons Attribution (CC-BY) license (http://creativecommons.org/licenses/by/4.0/). 